\title{
Article \\ Ovarian Masses-Applicable IOTA ADNEX Model versus Morphological Findings for Accurate Diagnosis and Treatment
}

\author{
Tudor Butureanu 1,2, ${ }^{\text {, Demetra Socolov }}{ }^{1,3,+} \mathbb{D}$, Daniela Roxana Matasariu ${ }^{1, *}$, Alexandra Ursache ${ }^{1}$, \\ Ana-Maria Apetrei ${ }^{2}$, Irina Dumitrascu 1,3, Ingrid Vasilache ${ }^{3}$ (D), Dorina Rudisteanu ${ }^{3}$, Vasile Lucian Boiculese 4 \\ and Ludmila Lozneanu 5,6
}

Citation: Butureanu, T.; Socolov, D.; Matasariu, D.R.; Ursache, A.; Apetrei, A.-M.; Dumitrascu, I.; Vasilache, I.; Rudisteanu, D.; Boiculese, V.L.; Lozneanu, L. Ovarian MassesApplicable IOTA ADNEX Model versus Morphological Findings for Accurate Diagnosis and Treatment. Appl. Sci. 2021, 11, 10789. https:// doi.org/10.3390/app112210789

Academic Editor: Carmen

Lacramioara Zamfir

Received: 14 September 2021

Accepted: 10 November 2021

Published: 15 November 2021

Publisher's Note: MDPI stays neutral with regard to jurisdictional claims in published maps and institutional affiliations.

Copyright: (C) 2021 by the authors Licensee MDPI, Basel, Switzerland. This article is an open access article distributed under the terms and conditions of the Creative Commons Attribution (CC BY) license (https:/ / creativecommons.org/licenses/by/ $4.0 /)$.
1 Departament of Mother and Child, "Grigore T. Popa" University of Medicine and Pharmacy, 700115 Iasi, Romania; tudorandreib@gmail.com (T.B.); demetrasocolov@gmail.com (D.S.); carpalecsandra@yahoo.com (A.U.); irina10021968@gmail.com (I.D.)

2 Department of Obstetrics and Gynecology, “Elena Doamna” Maternity Hospital, 700398 Iasi, Romania; ana.apetrei85@gmail.com

3 Department of Obstetrics and Gynecology, Hospital "Cuza-Voda", 700398 Iasi, Romania; tanasaingrid@yahoo.com (I.V.); rudisteanu.dorina@yahoo.com (D.R.)

4 Department of Preventive Medicine and Interdisciplinarity Medical Informatics and Biostatistics, "Grigore T. Popa" University of Medicine and Pharmacy, 700115 Iasi, Romania; lboiculese@gmail.com

5 Department of Morpho-Functional Sciences I-Histology, Pathology, "Grigore T. Popa” University of Medicine and Pharmacy, 700115 Iasi, Romania; ludmila.lozneanu@umfiasi.ro

6 Department of Pathology, "Sf. Spiridon" County Clinical Emergency Hospital, 700111 Iasi, Romania

* Correspondence: roxanamatasariu@yahoo.com

+ Equal contributions.

Abstract: A common problem in gynecological practice is the differential diagnosis of the ovarian masses. The clinician must apply the IOTA (International Ovarian Tumor Analysis) ADNEX (Assessment of Different Neoplasia in the Adnexa) model criteria to evaluate the risk of benign, borderline or malignant tumors. The aim of this study was to verify if the IOTA ADNEX model is a practical tool to be used before surgery and if there is a significant difference between IOTA ADNEX criteria and histological findings. A prospective single center study was performed between January 2017 and December 2019 in Obstetrics and Gynecology Hospital "Cuza-Voda", Iasi, Romania. The study included 230 patients between 17 and 74 years old diagnosed with persistent adnexal masses. We applied the IOTA ADNEX model protocol predicting the risk of benign, borderline or malignant masses. The golden standard remains the histological diagnosis of the surgically removed mass. The patients that had been diagnosed using ultrasonography with persistent adnexal masses between 30 and $291 \mathrm{~mm}$ were operated on in our clinic. In our study. the majority of patients had benign ovarian tumor mass, these being 223 (96.96\%) patients, from whom, according to IOTA ADNEX protocol, the correspondence was: $91.8-99.7 \%$ at risk of benign tumors, $0.3-4.5 \%$ at risk of borderline tumors and $0.3-8.2 \%$ at risk of malignant masses. Unexpected findings were obtained from the malignant group that included five patients $(2.17 \%)$ with the following correspondence: $96.1-99 \%$ at risk of benign tumors, $0.6-2.4 \%$ at risk of borderline tumors and $1-3.9 \%$ at risk of malignant masses. After applying the IOTA ADNEX model criteria, the patients with a suspicion of malignant disease were correctly guided towards surgical treatment in an oncological center. In our hospital, surgical treatment was only proposed to those patients with high suspicion of benign masses.

Keywords: adnexal mass; IOTA; ultrasound; histology; treatment

\section{Introduction}

An essential problem in gynecological practice is the differential diagnosis of ovarian tumors [1]. Ovarian masses are a frequent pathology found in our field of expertise [2,3]. Patients with ovarian malignant tumors are frequently diagnosed with this pathology in advanced stages, with limited treatment options and high mortality. It is therefore 
extremely important to have an accurate diagnosis before the surgical intervention to ensure better patient outcomes.

A pelvic ultrasound is the most important method for the diagnosis and characterization of an ovarian mass [2].

For the patients with an ovarian mass, the evaluation protocol requires the determination of the following cancer serum biomarkers: cancer antigen-125 (CA-125), carbohydrate antigen 19-9 (CA19-9), carcinoembryonic antigen (ACE) and human epididymis protein 4 (HE 4) [1]. A combination of these ovarian cancer serum biomarkers frequently used in general practice is ROMA (the Risk of Ovarian Malignancy Algorithm), a risk assessment combination of serum CA 125 and HE 4 [4-6].

It is important to have an experienced physician in the ultrasound evaluation of ovarian masses to lower the error margin and to make a correct differential diagnosis. It is true that a lot of tumors can be recognized from the beginning as benign (such as endometriosis cyst or teratoma) or malignant (advanced ovarian cancer) [1].

After a consensus conference in ultrasonography regarding the "investigation into designed reporting of adnexal masses that allows a better communication of findings and suggestions for follow-up", a team of specialists in gynecology, radiology and pathology established a system of criteria for a more accurate evaluation of ovarian mass [2,7]. This system is based on recognition patterns suggested by the IOTA (International Ovarian Tumor Analysis) [2,8].

Established in 2005, the IOTA simple rules can be used without the need to calculate, being very easy to apply and based only on ultrasound ovarian mass findings. This simple system can also be used by inexperienced clinicians. The IOTA ADNEX model, elaborated in 2014, is a more complex analysis of a patient with ovarian mass. It includes three clinical criteria (age, CA 125 serum level, and the type of center: oncological/other types of hospitals) and six ultrasound features (the maximum tumoral diameter in $\mathrm{mm}$, the maximum diameter of the solid component in $\mathrm{mm}$; more than 10 loculus, number of papillary projections, presence/absence of acoustic shadows and ascites) $[9,10]$.

Transvaginal ultrasonography is the most used investigation method for ovarian tumors [11]. Ultrasound examination must evaluate many of the ovarian mass characteristics, such as laterality (unilateral or bilateral), maximum diameter, echogenicity, wall thickness, cystic content, solid areas, septations, solid papillary projections, the presence of ascites and Doppler evaluation (peripheral or central blood flow and the presence or absence of septal vascularization) $[2,12]$. The suspicion of malignancy is based on the presence of a thick wall and septum, solid papillary projection, solid areas, the presence of ascites and central blood flow $[2,13,14]$.

The clinician must apply the IOTA criteria to make a difference between malignant, benign or borderline tumor potential. Furthermore, if there is a suspicion of a malignant mass, the patient will be directed to a specialized gynecologic oncology center. If there is a suspicion of a borderline or a malignant tumor, the patient must be directed to have a pelvic MRI exploration to evaluate the stage of malignancy [1,15-18].

Our intent is to identify imagistic and clinicomorphological approaches in order to improve the outcome of patients with an ovarian mass.

\section{Materials and Methods}

\subsection{Patients}

This was a prospective single center study (Obstetrics and Gynecology Hospital "Cuza Voda" Iasi, Romania) performed between 2017 and 2019, which included 230 patients, the age range being 17 to 74 years. Patient selection criteria included hypogastric pain, infertility, metrorrhagia, dysmenorrhea and dyspareunia.

At the outset of the medical investigations, the women had a clinical and paraclinical diagnosis of ovarian masses in the private sector through detailed history and using transvaginal and transabdominal ultrasonography. Following that, the cases with high suspicion of benign tumors were sent to our hospital for evaluation and surgery. In order 
to minimize bias, all the patients were evaluated by experienced specialists using the same ultrasound machine, the Voluson E8 equipped with IOTA ADNEX ${ }^{\mathrm{TM}}$ software.

Each patient signed a written informed consent. The informed consent was approved by the Commission for Medical Ethics of the University of Medicine Grigore T. Popa, Iasi (790/2017).

We collected all data pertaining to the patients that approached our clinic for ovarian masses. We observed the following morphological aspects:

1. Laterality (unilateral or bilateral);

2. Maximum diameter;

3. Echogenicity;

4. Wall thickness;

5. Cystic content;

6. Solid areas;

7. Septations;

8. Solid papillary projections;

9. The presence of ascites;

10. The Doppler evaluation [2,12].

From our hospital's electronic database, we retrieved data concerning the values of CA 125, the type of surgery and the histopathological results after the surgery.

We applied the IOTA ADNEX model protocol.

The preoperative classification system of adnexal masses is composed of 5 specific features for benign tumors (B-rules) and 5 specific features for malignant tumors (M-rules). These rules are useful in diagnosing patients with at least one persistent adnexal mass before surgical treatment.

Rules for predicting a benign tumor $\rightarrow$ B-rules:

- B1: unilocular cyst;

- B2: presence of solid components (largest diameter $<7 \mathrm{~mm}$ );

- B3: presence of acoustic shadowing;

- B4: smooth multilocular tumor with the largest diameter $<100 \mathrm{~mm}$;

- B5: no blood flow (color score 1).

Rules for predicting a malignant tumor $\rightarrow$ M-rules:

- M1: irregular solid tumor;

- M2: presence of ascites;

- M3: at least four papillary structures present;

- M4: irregular, multilocular, solid tumor with the largest diameter $>=100 \mathrm{~mm}$;

- M5: intensive blood flow (color score 4).

If one or more B-rules were present in the absence of M-rules, the ovarian masses were classified as benign. However, if one or more M-rules were present in the absence of B-rules, the mass was classified as malignant [2].

The ADNEX model is available at http:/ / www.iotagroup.org/adnexmodel/ (accessed on 17 June 2021) (Figure 1).

The IOTA ADNEX model is able to estimate the probability of an adnexal tumor of being benign, borderline or malignant $[11,19]$.

In the case the mass is suspected to be malignant (elevated CA-125, ascites, fixed pelvic mass, abdominal metastasis and/or increased IOTA ADNEX risk of malignancy), the patient should be directed to a gynecological oncology center.

The golden standard remains the histological diagnosis of the surgically removed tumor. 
IOTA - ADNEX model

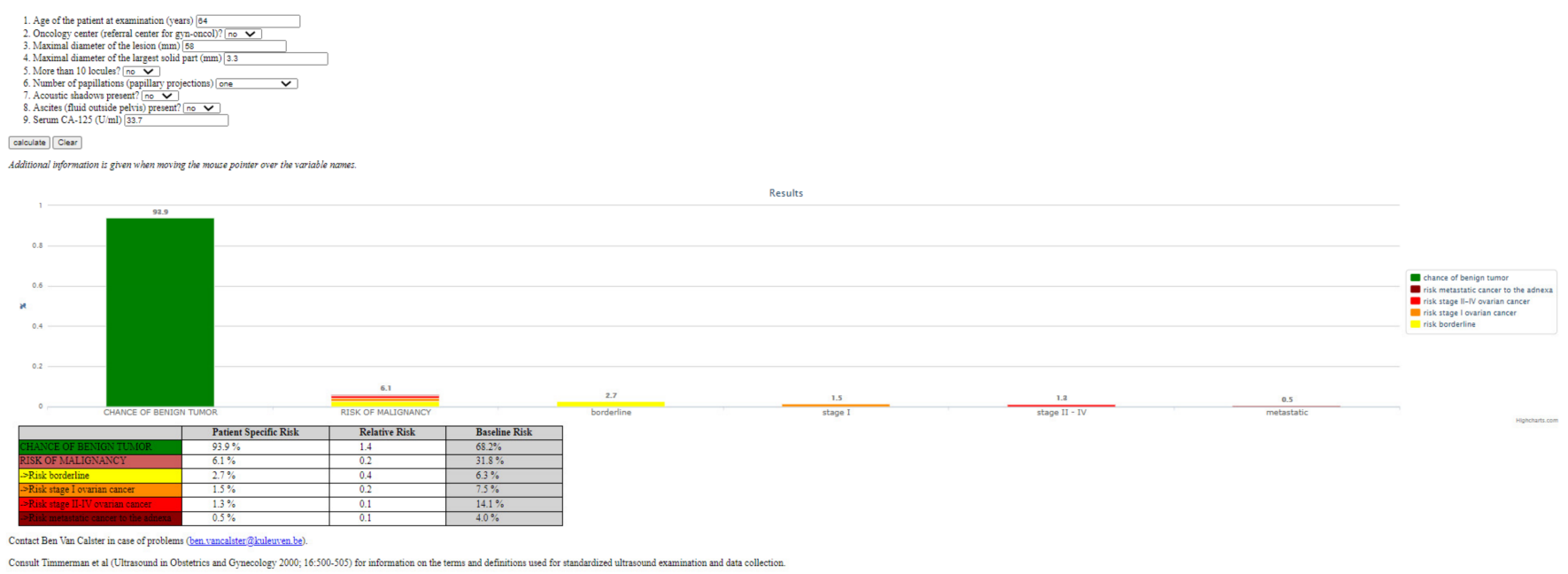

Figure 1. IOTA ADNEX model protocol used for the evaluation of a patient's specific risk.

\subsection{Statistical Analysis}

The set of data was examined with SPSS 18 application (PASW Statistics for Windows, SPSS Inc., Chicago, IL, USA). Descriptive statistics like frequencies for categorical data and mean, median, minimum and maximum were computed for ratio or interval scale, real number variable. Frequencies confidence intervals (95\%) were computed by a bootstrap method with 1000 samples. The inferential statistics for comparisons were made with a nonparametric Mann-Whitney test. The significance level was set to 0.05 for hypothesis testing.

\section{Results}

In our study, we examined patients with persistent adnexal masses between 30 and $291 \mathrm{~mm}$ (30-100 mm: 210 cases; 101-200 mm: 18 cases; 201-291 mm: 2 cases). Of the 230 patients with an ovarian mass, we detected $44(19.13 \%)$ cases in women with postmenopausal status, with the remaining $186(80.87 \%)$ being of fertile age.

Ultrasound examination revealed $27(11.74 \%)$ of these patients had bilateral ovarian masses, $34(14.78 \%)$ cases had solid components $<7 \mathrm{~mm}$ and only $5(2.17 \%)$ cases had solid components $>7 \mathrm{~mm}$.

By using the IOTA ADNEX model, our study detected that the rate of benign tumor masses was $91.8-99.7 \%$, the rate of borderline tumors represented $0.3-4.5 \%$ and the rate of malignant tumors was $0.3-8.2 \%$.

The final histopathological examination, after the surgery with the excision of the adnexal masses, showed a positive association in $223(96.96 \%)$ of the cases with benign tumors such as: endometrioma ( 64 cases: $28.70 \%$ ), mature cyst teratoma (27 cases: $12.11 \%$ ), hemorrhagic cyst (22 cases: 9.87\%), serous cystadenoma (74 cases: $33.18 \%$ ), mucinous cystadenoma (5 cases: $2.24 \%$ ), fibroma (16 cases: $7.17 \%$ ) and follicular cyst (15 cases: $6.73 \%$ ).

Borderline tumors (BOT) were found in only two cases $(0.87 \%)$ of women (28 and 63 years old). We have analyzed the percentages of cases (benign, borderline and malignant) and the confidence interval (Table 1). 
Table 1. Frequencies and confidence intervals of the pathological results.

\begin{tabular}{ccccc}
\hline \multirow{2}{*}{ Pathology } & \multirow{2}{*}{ No. of Cases } & Percent & \multicolumn{2}{c}{$\begin{array}{c}\text { 95\% } \\
\text { Confidence Interval }\end{array}$} \\
\cline { 4 - 5 } & & & Lower & Upper \\
\hline Benign & 223 & 96.96 & 94.78 & 98.7 \\
\hline Borderline & 2 & 0.87 & 0 & 2.17 \\
\hline Malign & 5 & 2.17 & 0.43 & 4.35 \\
\hline Total & 230 & 100 & 100 & 100 \\
\hline
\end{tabular}

We present a descriptive form for risk assessment percentages using the IOTA ADNEX model in the study group (Table 2).

Table 2. Risk assessment using the IOTA ADNEX model in the study groups.

\begin{tabular}{ccccc}
\hline \multirow{2}{*}{$\begin{array}{c}\text { IOTA ADNEX } \\
\text { Model }\end{array}$} & Measure & \multicolumn{3}{c}{ Histopathological Aspects } \\
\cline { 2 - 5 } & Mean & $98.26 \%$ & Borderline & Malign \\
\cline { 2 - 5 } Benign & Median & $98.60 \%$ & $98.95 \%$ & $97.40 \%$ \\
\cline { 2 - 5 } & Minimum & $77.30 \%$ & $98.95 \%$ & $96.80 \%$ \\
\cline { 2 - 5 } & Maximum & $99.70 \%$ & $99.40 \%$ & $96.10 \%$ \\
\hline \multirow{3}{*}{ Malign } & Mean & $1.75 \%$ & $1.05 \%$ & $99.00 \%$ \\
\cline { 2 - 5 } & Median & $1.40 \%$ & $1.05 \%$ & $2.60 \%$ \\
\cline { 2 - 5 } & Minimum & $0.30 \%$ & $0.60 \%$ & $3.20 \%$ \\
\hline \multirow{3}{*}{ Borderline } & Maximum & $22.70 \%$ & $1.50 \%$ & $3.00 \%$ \\
\cline { 2 - 4 } & Mean & $0.98 \%$ & $0.65 \%$ & $1.42 \%$ \\
\cline { 2 - 4 } & Median & $0.90 \%$ & $0.65 \%$ & $1.50 \%$ \\
\hline & Minimum & $0.30 \%$ & $0.50 \%$ & $0.60 \%$ \\
\hline
\end{tabular}

We have also compared the maximum dimension for the two groups and found a significance of effect size ( $p=0.029$, the same nonparametric Mann-Whitney U test). The mean $=154.8$, median $=148, \min =50$ and $\max =291$ for the histological malign type in relation to the mean $=63.7$, median $=60, \min =30$ and $\max =280$ for the non-malignant type. These differences show the importance of maximum dimension of the tumor in diagnosis.

Histologically confirmed malignant tumors were detected in only five $(2.17 \%)$ cases (two cases of HGSC (high-grade serous carcinoma), two cases of EC (endometrioid carcinoma) and one case of CC-OC (clear cells ovarian carcinoma)) (Figure 2), although the IOTA ADNEX model showed a malignant risk under $3.9 \%$ and a borderline tumor risk less than $2.4 \%$. Thus, we obtained $2.17 \%$ false negative results for malignancy. 
Case 1

47 years

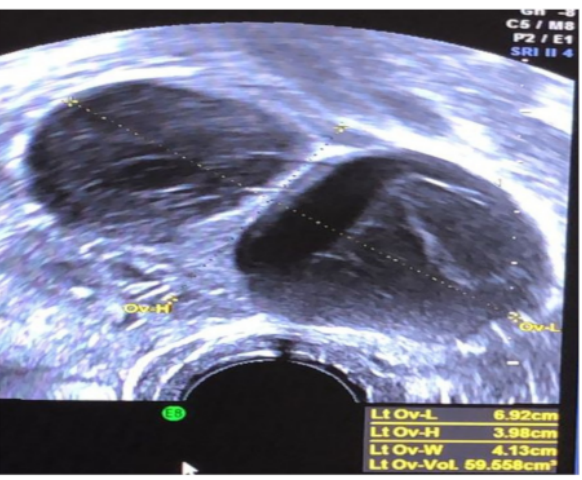

Hemorrhagic cyst

Case 2

38 years

Case 3

38 years

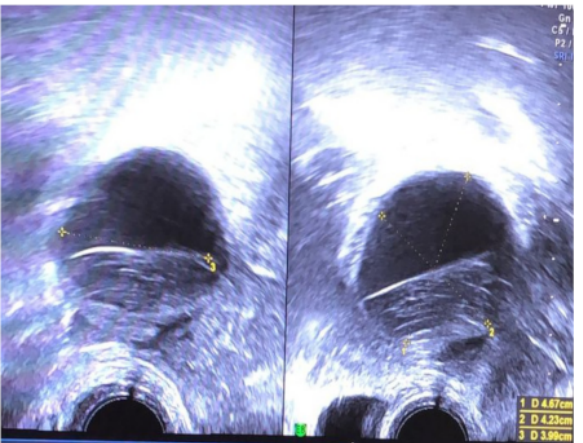

Hemorrhagic cyst

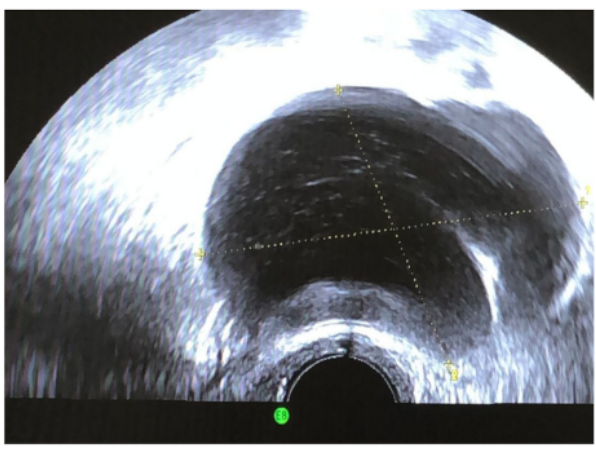

Hemorrhagic cyst

Case 4 56 years

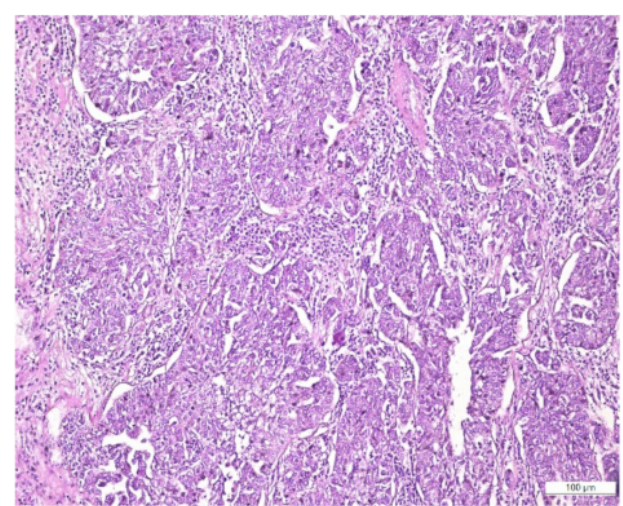

High-grade serous carcinoma: solid and papillary architecture with delicate lymphocytes infiltrating stroma (HEx10).

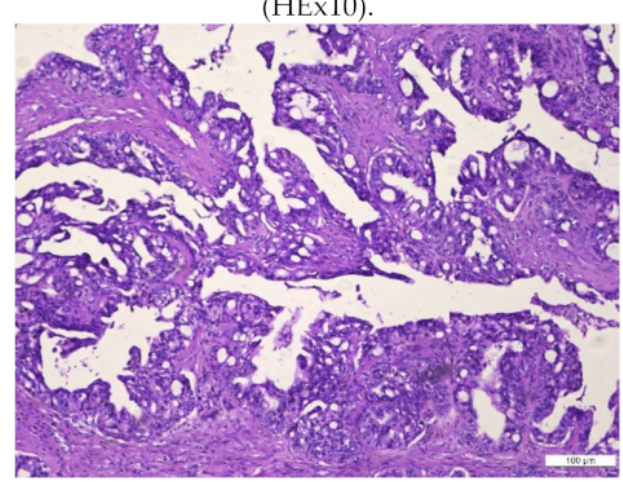

High-grade serous carcinoma: papillary growth with

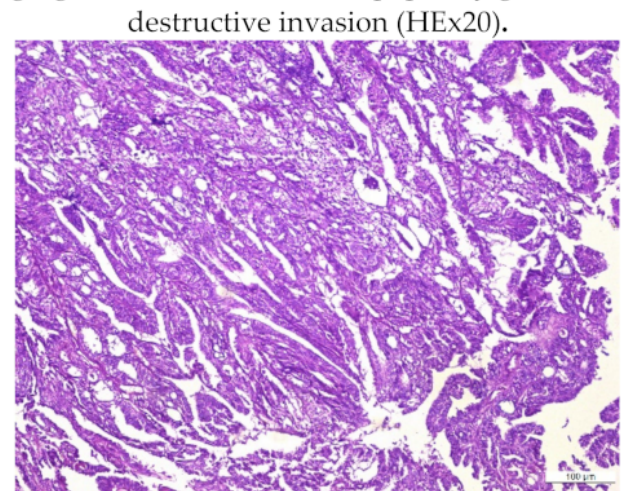

Endometrioid carcinoma: back-to-back gland with smooth luminal border (HEx10).

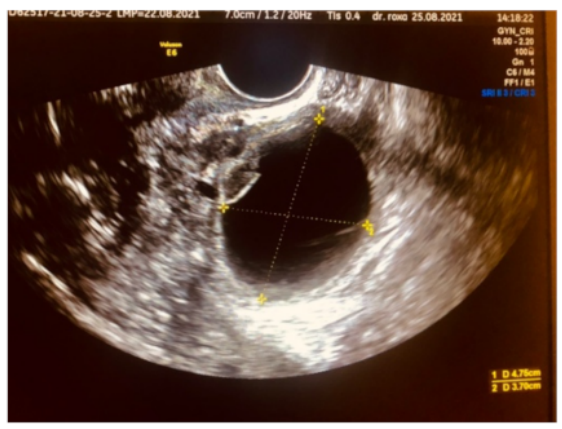

Serous cyst

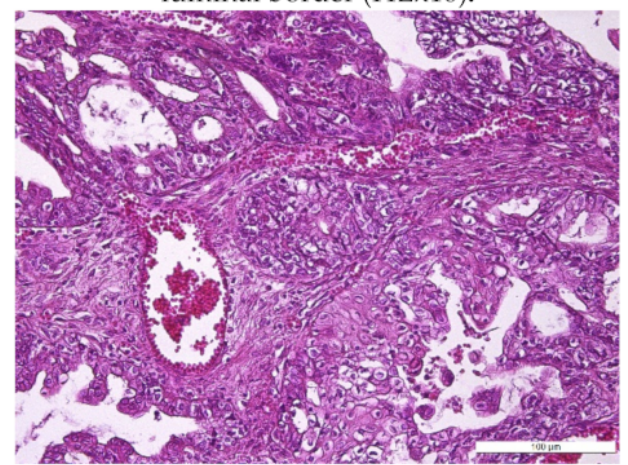

Endometrioid carcinoma: confluent arrangement of gland and solid growth with desmoplastic stroma (HEx20).

Figure 2. Cont. 


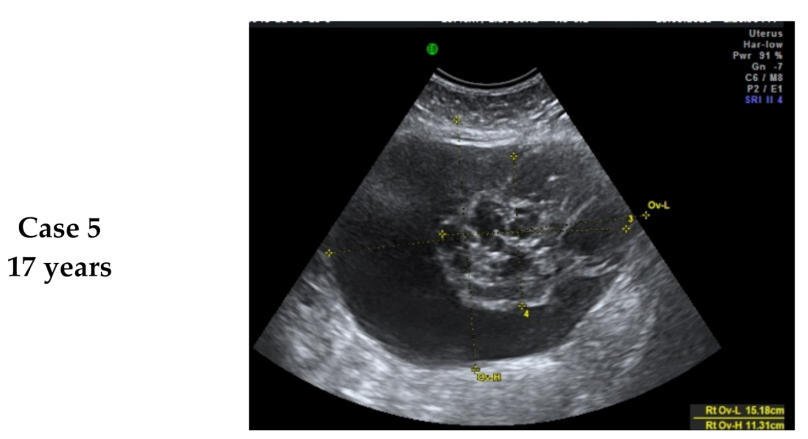

Serous cyst with solid component

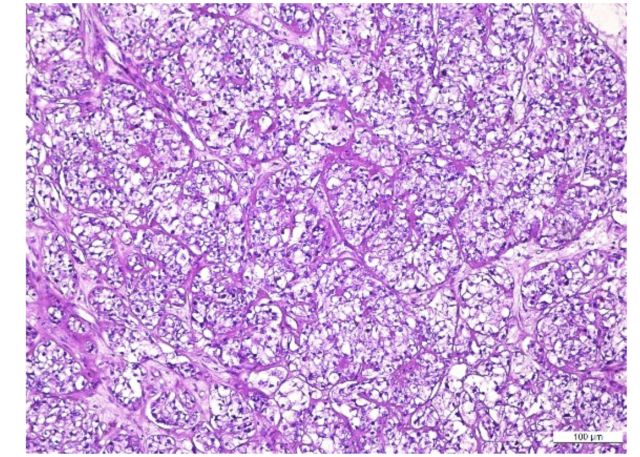

Clear cell carcinoma: solid growth separated by subtle stroma (HEx10).

Figure 2. Comparative imagistic and histology aspect in malignant cases: HGSC (high-grade serous carcinoma), EC (endometrioid carcinoma) and CC-OC (clear cell ovarian carcinoma).

\section{Discussion}

The advent of imagistic exploration has accelerated the possibility of an accurate ultrasound diagnosis. The main reason for attempting to establish a differential diagnosis between benign and malignant tumors is to correctly refer the patients with a malignant mass to an oncological center, where therapeutic results are clearly superior if treated by a gynecologic oncology team $[11,20,21]$.

Many patients with ovarian cancer are not diagnosed at an early stage due to a lack of symptoms, this aspect being responsible for its high mortality rate. More than $90 \%$ of the ovarian cancers could be managed successfully if a more specific diagnosis were possible in the early stage of cancer development. Not one of the serum biomarkers used to detect ovarian cancer showed enough high sensitivity and specificity to be detected in the early stage.

In many situations that concern patients with a persistent ovarian mass, especially in postmenopausal women, surgical treatment is recommended. Furthermore, the final diagnosis is based on the histological analysis, after the examination of the surgically removed tissue. The classification of ovarian tumors included in the study was based on the correlation of pathologic criteria of ovarian tumors 2020 according to the World Health Organization (WHO), considering the histopathological aspects that included a wide spectrum of malignancy aspects, such as the tumor growth, the arrangement of glands, the morphology of the lining epithelium, the pattern of invasion and the stromal characteristics [22].

Previously, the ultrasound examination alone, or the combinations between ultrasound correlated with serum biomarkers, seemed to be the best modalities to detect ovarian cancer and to distinguish between malignant or benign ovarian masses. In addition, the ultrasound can influence the decisional strategy of surgical treatment. It has become clear that transvaginal sonography has a sensitivity of $<90 \%$ for early ovarian cancer and a specificity of $94-99 \%[11,20,23]$.

In assessing the malignancy risk of an ovarian mass, there are a lot of scoring systems based on ultrasound. The IOTA group proposes two original models to predict the risk of malignancy in an ovarian mass: the ultrasound Simple Rules and the ADNEX model [11,24].

In order to identify the accuracy of imagistic evaluation, we compared the results obtained according to the IOTA criteria analysis of the ovarian mass versus morphological aspects of the lesion.

We classified the tumors according to the IOTA ADNEX model as being benign, borderline and malign. Afterwards, we compared the results to the pathological examination. For comparison of the IOTA ADNEX model chance of a benign tumor, we used a non- 
parametric test because we did not have the statistical power to verify the normality of the data. Our results showed that the averages are close but so are the medians.

The results of our two-year follow-up study of ovarian mass showed that the IOTA ADNEX model provides more accurate results than the ultrasound examination alone in differentiating between benign and malignant adnexal masses. It also strongly correlates with the histopathological findings having a rate of error of only $2.17 \%$. In only $5(2,17 \%)$ of the 230 of cases that we analyzed, the IOTA ADNEX model estimated a very low risk of malignancy, and the histopathological examination revealed the presence of a malignant mass.

We compared the IOTA ADNEX model chance of a malignant tumor for the groups defined by malign versus the non-malignant histological aspect. The Mann-Whitney $U$ test shows no statistical significance $(p=0.123)$ because the data are complementary. Thus, we have no statistically confirmed differences.

When we compared the IOTA ADNEX model chance of a benign tumor for the groups defined by malign versus the non-malignant histological aspect, the Mann-Whitney $U$ test also showed no statistical significance $(p=0.123)$. Thus, this score is no different when comparing the malignant versus the non-malignant group.

When we compared the maximum size of the tumor for the two groups, we obtained statistical significance $(p=0.029)$, the maximum size being over two times larger for malignant tumors.

Taking into consideration the fact that all the patients with highly suggestive malignancy ultrasound evaluations were directly referred to a gynecologic oncology center from the private sector, the IOTA ADNEX model is more specific and sensitive than ultrasound exploration alone in detecting early-stage ovarian cancer. The rate of error of this model is very low, and the accuracy tends to be closer to $98 \%$ if we exclude patients with in situ squamous carcinoma with microinvasion.

The false negative evaluation of the five ovarian masses as benign tumor does not seem to be correlated with the patient's age or with their fertile status. Further, these changes were independent of menopausal status.

This method is considered to be a highly useful tool in developing countries that need to be extremely effective in triaging patients to offer cost-efficient management. These changes were independent of menopausal status. Further, we found that the risk of malignancy was $3.9 \%$ and under $2.4 \%$ for a borderline tumor.

The ACOG (American College of Obstetricians and Gynecologists) also suggests that the IOTA ADNEX model predicts the risk of a specific type of adnexal mass with high accuracy and can therefore offer better management for patients with ovarian tumors $[25,26]$.

The IOTA ADNEX model is available in two versions: with or without the inclusion of the CA-125 value. Van Calster B. et al., 2014, concluded that CA-125 is a nonspecific marker in the differentiation between benign or malignant adnexal masses $[10,27]$. Erdogan Nohuz, 2018, used the IOTA ADNEX model algorithm in 107 patients over 43 years old. The algorithm proved to be very useful in distinguishing between benign and malignant tumors. An ultrasound examination can be used by inexperienced sonographers and may help them to correctly evaluate the findings and classify them as presumed benign or malignant, representing a useful tool for sorting these patients for further, more specific explorations [28].

As we showed in our study, Szubert et al., 2016, demonstrated that the results obtained using this IOTA ADNEX model were highly accurate and can be used for the differential diagnosis of ovarian masses [29].

However, our study had some limitations. First, the small number of patients included in our study and, second, the lack of evidence of the number of patients that were evaluated in the private sector that might have been directly referred to an oncological center without being evaluated in our hospital beforehand. In this context, the imagistic mechanism by which some parameters cause radically different results (malignant lesion identified on 
surgical samples versus the IOTA ADNEX criteria) has not been shown in all cases, as in our case.

However, one of the strong points to support our findings is the fact that there are few prospective studies in the literature that evaluate the accuracy of the IOTA ADNEX model evaluation of ovarian masses. This is the main reason we want to embark on a larger prospective study with the help of our gynecologic oncology colleagues to better evaluate the accuracy of this important scoring system and help implement this protocol of evaluation in our country.

Some ultrasound features may cause radically different results, such as a malignant lesion being identified on surgical samples as benign or borderline through IOTA criteria risk assessment. In our case, five $(2.17 \%)$ of these patients had malignant tumors on the histological exam, associated with changes that were suitable for benign or borderline lesions according to the IOTA ADNEX criteria. These findings were consistent with the results of Quaranta et al. 2020 that estimated a rate of $1 \%$ of false negatives [30]. Our results in these five cases as "probably benign", according to the IOTA ADNEX model, were probably due to the fact that the tumors were of first-stage ovarian malignancy with very small cancer foci in the cystic masses. As expected and proved in literature, the IOTA ADNEX model has a more accurate predictive value for stage-II-IV ovarian cancers and lower predictive values for borderline tumors and first-stage ovarian cancer [31]. The risk of malignancy of these five cases with false negative IOTA ADNEX model evaluation was a very low $<3.9 \%$. To improve the accuracy, we might use a smaller cutoff value for the IOTA ADNEX model. We can consider a cutoff value of over 3\% for malignancy in association with a risk of over $1.5 \%$ for borderline tumors in clinical practice, but with the disadvantage of invasive procedures being carried out in a larger number of patients with benign ovarian masses that could be managed with conservative or follow-up treatment.

However, using the IOTA ADNEX model, we confirmed the efficiency of this model in another $223(96.96 \%)$ cases with benign tumors. Hence, the IOTA ADNEX protocol on ovary mass generally has favorable clinical and pathological correlations. It is important to determine if the imagistic changes can predict morphology. In literature, the rate of false positive evaluations seems to be larger than false negative ones [30]. Therefore, clarifying the role of the correct imagistic algorithm and its parameters by clear criteria, which may include the IOTA ADNEX model, remains an important issue in the field.

\section{Conclusions}

Our study shows that the IOTA ADNEX model is able to differentiate more accurately between benign, borderline and malignant ovarian tumors. One major reason IOTA ADNEX model rules have not been widely implemented in Romania is the lack of validation for them in our country. IOTA Simple Rules are used in countries such as Belgium, France, Italy, Sweden and the United Kingdom, while the IOTA ADNEX model is implemented in many countries such as Belgium, Canada, China, Czech Republic, France, Italy, Poland, Spain, Sweden and the United Kingdom.

Therefore, it improves the decisions of patient triage and management, thus reducing the morbidity and mortality associated with adnexal pathology. These methods can be used even in the absence of an experienced clinician, with successful management and better patient outcomes.

ADNEX is available for free online as an application on smartphones http:/ /www. iotagroup.org/adnexmodel/ (accessed on 17 June 2021) and, in addition to this, newer ultrasound machines have been incorporating this algorithm.

Author Contributions: Conceptualization, D.R.M., A.U., L.L. and D.S.; methodology, D.R.M. and L.L.; software, V.L.B., A.-M.A., I.V. and I.D.; validation, L.L. and D.S.; investigation, T.B. and D.S.; writing-original draft preparation, D.R.M., L.L. and D.R.; writing-review and editing, D.R.M. and L.L.; supervision, D.R.M., L.L. and D.S. All authors have read and agreed to the published version of the manuscript. 
Funding: No funding was received for this study.

Institutional Review Board Statement: The study was conducted according to the guidelines of the Declaration of Helsinki, and the protocol was approved by the Ethics Committee of the "Grigore T. Popa" University of Medicine and Pharmacy, Iasi (790/2017).

Informed Consent Statement: Informed consent was obtained from all subjects involved in the study.

Data Availability Statement: The data used to support the findings of this study are available upon request to the authors.

Conflicts of Interest: The authors declare no conflict of interest.

\section{References}

1. Szubert, S.; Szpurek, D.; Wójtowicz, A.; Żywica, P.; Stukan, M.; Sajdak, S.; Jabłonski, S.; Wicherek, Ł.; Moszyński, R. Performance of Selected Models for Predicting Malignancy in Ovarian Tumors in Relation to the Degree of Diagnostic Uncertainty by Subjective Assessment with Ultrasound. J. Ultrasound Med. 2019, 39, 939-947. [CrossRef] [PubMed]

2. $\quad$ Basha, M.A.A.; Refaat, R.; Ibrahim, S.A.; Madkour, N.M.; Awad, A.M.; Mohamed, E.M.; El Sammak, A.A.; Zaitoun, M.M.A.; Dawoud, H.A.; Khamis, M.E.M.; et al. Gynecology Imaging Reporting and Data System (GI-RADS): Diagnostic Performance and Inter-Reviewer Agreement. Eur. Radiol. 2019, 29, 5981-5990. [CrossRef] [PubMed]

3. Harris, R.D.; Javitt, M.C.; Glanc, P.; Brown, D.L.; Dubinsky, T.; Harisinghani, M.G.; Khati, N.J.; Kim, Y.B.; Mitchell, D.G.; Pandharipande, P.V.; et al. ACR Appropriateness Criteria ${ }^{\circledR} C$ linically Suspected Adnexal Mass. Ultrasound Q. 2013, $29,79-86$. [CrossRef]

4. Jeong, S.Y.; Park, B.K.; Lee, Y.Y.; Kim, T.J. Validation of IOTA-ADNEX Model in Discriminating Characteristics of Adnexal Masses: A Comparison with Subjective Assessment. J. Clin. Med. 2020, 9, 2010. [CrossRef] [PubMed]

5. Geomini, P.; Kruitwagen, R.F.; Bremer, G.L.; Cnossen, J.; Mol, B.W. The Accuracy of Risk Scores in Predicting Ovarian Malignancy. Obstet. Gynecol. 2009, 113, 384-394. [CrossRef]

6. Moore, R.G.; McMeekin, D.S.; Brown, A.K.; DiSilvestro, P.; Miller, M.C.; Allard, W.J.; Gajewski, W.; Kurman, R.; Bast, R.C., Jr.; Skates, S.J. A novel multiple marker bioassay utilizing HE4 and CA125 for the prediction of ovarian cancer in patients with a pelvic mass. Gynecol. Oncol. 2009, 112, 40-46. [CrossRef]

7. Levine, D.; Brown, D.L.; Andreotti, R.F.; Benacerraf, B.; Benson, C.B.; Brewster, W.R.; Coleman, B.; Depriest, P.; Doubilet, P.M.; Goldstein, S.R.; et al. Management of Asymptomatic Ovarian and Other Adnexal Cysts Imaged at US: Society of Radiologists in Ultrasound Consensus Conference Statement. Radiology 2010, 256, 943-954. [CrossRef]

8. Orozco, F.R.; Peces, R.A.; Llanos, M.C.; Martines, M.A.; Machado, L.; Nieto, D.A. Clinical application of the gynecologic imaging reporting and data system (GI-RADS) for the evaluation of adnexal masses. SM J. Gynecol. Obstet. 2015, 1, $1009-1012$.

9. Viora, E.; Piovano, E.; Poma, C.B.; Cotrino, I.; Castiglione, A.; Cavallero, C.; Sciarrone, A.; Bastenero, S.; Iskra, L.; Zola, P. The ADNEX model to triage adnexal masses: An external validation study and comparison with the IOTA two-step strategy and subjective assessment by an experienced ultrasound operator. Eur. J. Obstet. Gynecol. Reprod. Biol. 2020, 247, 207-211. [CrossRef] [PubMed]

10. Van Calster, B.; Van Hoorde, K.; Valentin, L.; Testa, A.C.; Fischerova, D.; Van Holsbeke, C.; Savelli, L.; Franchi, D.; Epstein, E.; Kaijser, J.; et al. Evaluating the Risk of Ovarian Cancer before Surgery Using the ADNEX Model to Differentiate between Benign, Borderline, Early and Advanced Stage Invasive, and Secondary Metastatic Tumours: Prospective Multicentre Diagnostic Study. BMJ 2014, 349, g5920. [CrossRef]

11. Abramowicz, J.S.; Timmerman, D. Ovarian Mass-Differentiating Benign from Malignant: The Value of the International Ovarian Tumor Analysis Ultrasound Rules. Am. J. Obstet. Gynecol. 2017, 217, 652-660. [CrossRef]

12. Alcázar, J.L.; Pascual, M.A.; Graupera, B.; Aubá, M.; Errasti, T.; Olartecoechea, B.; Ruiz-Zambrana, A.; Hereter, L.; Ajossa, S.; Guerriero, S. External Validation of IOTA Simple Descriptors and Simple Rules for Classifying Adnexal Masses. Ultrasound Obstet. Gynecol. 2016, 48, 397-402. [CrossRef] [PubMed]

13. Timmerman, D.; Valentin, L.; Bourne, T.H.; Collins, W.P.; Verrelst, H.; Vergote, I.; International Ovarian Tumor Analysis (IOTA) Group. Terms, Definitions and Measurements to Describe the Sonographic Features of Adnexal Tumors: A Consensus Opinion from the International Ovarian Tumor Analysis (IOTA) Group. Ultrasound Obstet. Gynecol. 2000, 16, 500-505. [CrossRef] [PubMed]

14. Alcázar, J.L.; Errasti, T.; Laparte, C.; Jurado, M.; López-García, G. Assessment of a New Logistic Model in the Preoperative Evaluation of Adnexal Masses. J. Ultrasound. Med. 2001, 20, 841-848. [CrossRef]

15. Van Gorp, T.; Veldman, J.; Van Calster, B.; Cadron, I.; Leunen, K.; Amant, F.; Timmerman, D.; Vergote, I. Subjective Assessment by Ultrasound Is Superior to the Risk of Malignancy Index (RMI) or the Risk of Ovarian Malignancy Algorithm (ROMA) in Discriminating Benign from Malignant Adnexal Masses. Eur. J. Cancer 2012, 48, 1649-1656. [CrossRef]

16. Moore, R.G.; Miller, M.C.; Disilvestro, P.; Landrum, L.M.; Gajewski, W.; Ball, J.J.; Skates, S.J. Evaluation of the Diagnostic Accuracy of the Risk of Ovarian Malignancy Algorithm in Women with a Pelvic Mass. Obstet. Gynecol. 2011, 118, 280-288. [CrossRef]

17. Sayasneh, A.; Kaijser, J.; Preisler, J.; Smith, A.A.; Raslan, F.; Johnson, S.; Husicka, R.; Ferrara, L.; Stalder, C.; Ghaem-Maghami, S.; et al. Accuracy of Ultrasonography Performed by Examiners with Varied Training and Experience in Predicting Specific Pathology of Adnexal Masses. Ultrasound Obstet. Gynecol. 2015, 45, 605-612. [CrossRef] 
18. Wynants, L.; Timmerman, D.; Verbakel, J.Y.; Testa, A.; Savelli, L.; Fischerova, D.; Franchi, D.; Van Holsbeke, C.; Epstein, E.; Froyman, W.; et al. Clinical Utility of Risk Models to Refer Patients with Adnexal Masses to Specialized Oncology Care: Multicenter External Validation Using Decision Curve Analysis. Clin. Cancer Res. 2017, 23, 5082-5090. [CrossRef] [PubMed]

19. Sayasneh, A.; Ferrara, L.; De Cock, B.; Saso, S.; Al-Memar, M.; Johnson, S.; Kaijser, J.; Carvalho, J.; Husicka, R.; Smith, A.; et al. Evaluating the Risk of Ovarian Cancer before Surgery Using the ADNEX Model: A Multicentre External Validation Study. Br. J. Cancer 2016, 115, 542-548. [CrossRef]

20. Woo, Y.L.; Kyrgiou, M.; Bryant, A.; Everett, T.; Dickinson, H.O. Centralisation of Services for Gynaecological Cancer. Cochrane Database Syst. Rev. 2012, CD007945. [CrossRef]

21. Van Nagell, J.R.; Miller, R.W. Evaluation and Management of Ultrasonographically Detected Ovarian Tumors in Asymptomatic Women. Obstet. Gynecol. 2016, 127, 848-858. [CrossRef] [PubMed]

22. Cheung, A.N.; Ellenson, L.H.; Gilks, C.B.; Kim, K.R.; Kong, C.S.; Lax, S.F.; Longacre, T.A.; Malpica, A.; McCluggage, W.G.; Oliva, E.; et al. Tumors of the Ovary. In Female Genital Tumours, 5th ed.; WHO Classification of Tumours Editorial Board, Ed.; International Agency for Research on Cancer: Lyon, France, 2020; Volume 4.

23. Bourne, T.H.; Campbell, S.; Reynolds, K.M.; Whitehead, M.I.; Hampson, J.; Royston, P.; Crayford, T.J.; Collins, W.P. Screening for Early Familial Ovarian Cancer with Transvaginal Ultrasonography and Colour Blood Flow Imaging. BMJ 1993, 306, $1025-1029$. [CrossRef]

24. Timmerman, D.; Van Calster, B.; Testa, A.; Savelli, L.; Fischerova, D.; Froyman, W.; Wynants, L.; Van Holsbeke, C.; Epstein, E.; Franchi, D.; et al. Predicting the Risk of Malignancy in Adnexal Masses Based on the Simple Rules from the International Ovarian Tumor Analysis Group. Am. J. Obstet. Gynecol. 2016, 214, 424-437. [CrossRef]

25. Meys, E.M.J.; Jeelof, L.S.; Achten, N.M.J.; Slangen, B.F.M.; Lambrechts, S.; Kruitwagen, R.F.P.M.; Van Gorp, T. Estimating Risk of Malignancy in Adnexal Masses: External Validation of the ADNEX Model and Comparison with Other Frequently Used Ultrasound Methods. Ultrasound Obstet. Gynecol. 2017, 49, 784-792. [CrossRef]

26. Committee Opinion No. 477: The Role of the Obstetrician-Gynecologist in the Early Detection of Epithelial Ovarian Cancer. Obstet. Gynecol. 2011, 117, 742-746. [CrossRef]

27. Froyman, W.; Timmerman, D. Methods of Assessing Ovarian Masses: International Ovarian Tumor Analysis Approach. Obstet. Gynecol. Clin. N. Am. 2019, 46, 625-641. [CrossRef] [PubMed]

28. Nohuz, E.; De Simone, L.; Chêne, G. Reliability of IOTA Score and ADNEX Model in the Screening of Ovarian Malignancy in Postmenopausal Women. J. Gynecol. Obstet. Hum. Reprod. 2019, 48, 103-107. [CrossRef] [PubMed]

29. Szubert, S.; Wojtowicz, A.; Moszynski, R.; Zywica, P.; Dyczkowski, K.; Stachowiak, A.; Sajdak, S.; Szpurek, D.; Alcazar, J.L. External Validation of the IOTA ADNEX Model Performed by Two Independent Gynecologic Centers. Gynecol. Oncol. 2016, 142, 490-495. [CrossRef]

30. Quaranta, M.; Nath, R.; Mehra, G.; Diab, Y.; Sayasneh, A. Surgery of Benign Ovarian Masses by a Gynecological Cancer Surgeon: A Cohort Study in a Tertiary Cancer Centre. Cureus 2020, 12, e9201. [CrossRef] [PubMed]

31. Van Calster, B.; Van Hoorde, K.; Froyman, W.; Kaijser, J.; Wynants, L.; Landolfo, C.; Anthoulakis, C.; Vergote, I.; Bourne, T.; Timmerman, D. Practical guidance for applying the ADNEX model from the IOTA group to discriminate between different subtypes of adnexal tumors. Facts Views Vis. Obgyn. 2015, 7, 32-41. 